$\underline{\text { Preprint typeset in JHEP style - PAPER VERSION }}$

Edinburgh 2008/18

MKPH-T-08-07

SHEP-08-15

\title{
The pion's electromagnetic form factor at small momentum transfer in full lattice QCD
}

\author{
P.A. Boyle ${ }^{a}$, J.M. Flynn ${ }^{b}$, A. Jüttner ${ }^{c}$, C. Kelly ${ }^{a}$, H. Pedroso de Lima ${ }^{b}$, \\ C.M. Maynard ${ }^{d}$, C.T. Sachrajda ${ }^{b}$, J.M. Zanotti ${ }^{a}$ \\ ${ }^{a}$ SUPA, School of Physics, The University of Edinburgh, \\ Edinburgh, EH9 3JZ, UK \\ ${ }^{b}$ School of Physics and Astronomy, University of Southampton, \\ Southampton, SO17 1BJ, UK \\ ${ }^{c}$ Institut für Kernphysik, Johannes-Gutenberg Universität Mainz, \\ D-55116 Mainz, Germany \\ ${ }^{d}$ EPCC, School of Physics, The University of Edinburgh, \\ Edinburgh, EH9 3JZ, UK
}

\section{RBC and UKQCD Collaborations}

ABstract: We compute the electromagnetic form factor of a "pion" with mass $m_{\pi}=$ $330 \mathrm{MeV}$ at low values of $Q^{2} \equiv-q^{2}$, where $q$ is the momentum transfer. The computations are performed in a lattice simulation using an ensemble of the RBC/UKQCD collaboration's gauge configurations with Domain Wall Fermions and the Iwasaki gauge action with an inverse lattice spacing of $1.73(3) \mathrm{GeV}$. In order to be able to reach low momentum transfers we use partially twisted boundary conditions using the techniques we have developed and tested earlier. For the pion of mass $330 \mathrm{MeV}$ we find a charge radius given by $\left\langle r_{\pi}^{2}\right\rangle_{330 \mathrm{MeV}}=0.354(31) \mathrm{fm}^{2}$ which, using NLO SU(2) chiral perturbation theory, extrapolates to a value of $\left\langle r_{\pi}^{2}\right\rangle=0.418(31) \mathrm{fm}^{2}$ for a physical pion, in agreement with the experimentally determined result. We confirm that there is a significant reduction in computational cost when using propagators computed from a single time-slice stochastic source compared to using those with a point source; for $m_{\pi}=330 \mathrm{MeV}$ and volume $(2.74 \mathrm{fm})^{3}$ we find the reduction is approximately a factor of 12 .

Keywords: Lattice QCD, Nonperturbative Effects, Pion Physics, Electromagnetic Processes and Properties. 


\section{Contents}

1. Introduction 1

2. Twisted boundary conditions and the form factor at small $Q^{2} \quad 2$

3. The computation and results 4

3.1 Correlation functions 4

3.2 Parameters of the simulation 5

3.3 Three point functions from noise source propagators 6

3.4 Electromagnetic form factor of a pion with $\boldsymbol{m}_{\boldsymbol{\pi}}=\mathbf{3 3 0} \mathrm{MeV} \quad 8$

3.5 Comparison of the cost of using point source and $\mathbb{Z}(2)$-wall source propagators 10

4. Electromagnetic form factor of a physical pion $\quad 11$

4.1 Chiral perturbation theory for the pion electromagnetic form factor 11

$\begin{array}{lll}4.2 & \text { Results for the physical pion } & 14\end{array}$

5. Summary and conclusions $\quad 16$

\section{Introduction}

In this paper we compute the electromagnetic form factor of a "pion" with mass $m_{\pi}=$ $330 \mathrm{MeV}$ at low values of $Q^{2} \equiv-q^{2}$, where $q$ is the momentum transfer. The computations are performed in a lattice simulation using an ensemble of the RBC/UKQCD collaboration's gauge configurations with Domain Wall Fermions and the Iwasaki gauge action with an inverse lattice spacing of $1.73(3) \mathrm{GeV}$ (see section 3.2 for brief details of the simulation and ref. [1] for a full discussion ${ }^{1}$ ). The action has good chiral and flavour symmetries and as demonstrated in ref. [1] a mass of $330 \mathrm{MeV}$ is well within the regime where NLO SU(2) chiral perturbation theory holds for other physical quantities, such as the meson masses, decay constants and the kaon's bag parameter.

In order to be able to reach low momentum transfers we employ partially twisted boundary conditions using the techniques developed and tested in ref. [2]. Previous lattice computations have used quarks satisfying periodic boundary conditions and therefore obtained form factors at much larger values of $Q^{2}$ (see however, the preliminary study

\footnotetext{
${ }^{1}$ In ref. [1] the pion mass corresponding to the bare quark mass used in the present study was found to be $331(6) \mathrm{MeV}$, where the error is dominated by the uncertainty in the lattice spacing. In the text we refer to this meson as having a mass of $330 \mathrm{MeV}$, while in the analysis we treat the fluctuations in the mass using a jackknife procedure.
} 
with twisted boundary conditions presented by the European Twisted Mass Collaboration (ETMC) [3]). For the pion with $m_{\pi}=330 \mathrm{MeV}$ we find for the charge radius, $\left\langle r_{\pi}^{2}\right\rangle_{330 \mathrm{MeV}}=0.354(31) \mathrm{fm}^{2}$. We then use NLO chiral perturbation theory to obtain the form factor and charge radius of a physical pion, finding

$$
\left\langle r_{\pi}^{2}\right\rangle=0.418(31) \mathrm{fm}^{2}
$$

in agreement with the experimentally determined value.

The power of the technique is demonstrated in fig. 2 where the data points are obtained from our simulation. The dashed vertical line is the minimum value of $Q^{2}\left(Q_{\mathrm{min}}^{2}\right)$ which is accessible with periodic boundary conditions. From the figure we see that the form factor can be obtained at arbitrarily small values of $Q^{2}$ and also that the results obtained with twisted boundary conditions join smoothly onto those obtained by performing the Fourier sum in the conventional way (i.e. onto the data point on the dashed line). In this paper we focus on the pion's electromagnetic form factor, but we anticipate that the technique used here will also have important applications to the calculation of other flavour non-singlet form factors at arbitrary values of momentum transfer, such as those which appear in $K_{\ell 3}$ semileptonic decays [2].

For this calculation we use propagators generated from a single time-slice stochastic source in addition to standard point source propagators. We compare the cost, obtaining similar errors for the pion mass, the normalization constant of the vector current, $Z_{V}$, and the pion's electromagnetic form factor at $Q_{\min }^{2}$, finding, for $m_{\pi}=330 \mathrm{MeV}$ and volume $(2.74 \mathrm{fm})^{3}$, a gain of approximately a factor of 12 in favour of the noise source propagators. A gain was also found in the preliminary study presented by the ETMC collaboration in [3] and in the recent publication by the UKQCD collaboration [4].

The plan for the remainder of this paper is as follows. In the next section we briefly review the use of partially twisted boundary conditions to compute the form factor at values of $Q^{2}$ which are inaccessible with periodic boundary conditions [2]. The details of the computation, the parameters of the simulation and the results for the form factor for the $330 \mathrm{MeV}$ pion are presented in section 3. The use of noise source propagators to evaluate the three point correlation functions from which the form factor is obtained is briefly described in section 3.3 and a comparison of the relative cost of using point source and noise source propagators to obtain results with the same statistical error is given in section 3.5. The use of NLO chiral perturbation theory to obtain the form factor and charge radius for a physical pion from that with mass $330 \mathrm{MeV}$ is described and performed in section 4 . Finally, in section 5 we present our conclusions.

\section{Twisted boundary conditions and the form factor at small $Q^{2}$}

The electromagnetic form factor of the pion, $f^{\pi \pi}\left(q^{2}\right)$, is defined by the matrix element

$$
\left\langle\pi^{+}\left(p^{\prime}\right)\left|V_{\mu}\right| \pi^{+}(p)\right\rangle=\left(p+p^{\prime}\right)_{\mu} f^{\pi \pi}\left(q^{2}\right), \quad \text { where } \quad q^{2}=-Q^{2}=\left(p-p^{\prime}\right)^{2}
$$




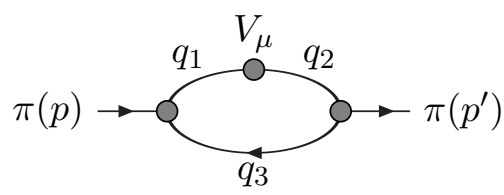

Figure 1: Sketch of the valence quark flow in the electromagnetic form factor of the pion. There is a similar contribution in which the current is on the antiquark line and the spectator is a quark.

and $V_{\mu}=\frac{2}{3} \bar{u} \gamma_{\mu} u-\frac{1}{3} \bar{d} \gamma_{\mu} d$ is the electromagnetic current. In a finite volume with periodic boundary conditions for the quark fields, the accessible pion momenta are given by

$$
p=\left(E_{n}, \vec{p}_{\vec{n}}\right)=\left(E_{n},(2 \pi / L) \vec{n}\right) \quad \text { and } \quad p^{\prime}=\left(E_{n^{\prime}}, \vec{p}_{\vec{n}^{\prime}}\right)=\left(E_{n^{\prime}},(2 \pi / L) \vec{n}^{\prime}\right)
$$

where $\vec{n}$ and $\vec{n}^{\prime}$ are vectors of integers, $L$ is the spatial extent of the lattice and $E_{n}$ and $E_{n^{\prime}}$ are the corresponding energies $\left(E_{n}^{2}=m_{\pi}^{2}+(2 \pi / L)^{2}|\vec{n}|^{2}\right.$ and $E_{n^{\prime}}^{2}=m_{\pi}^{2}+(2 \pi / L)^{2}\left|\vec{n}^{\prime}\right|^{2}$, where $m_{\pi}$ is the mass of the pion), so that $q^{2}$ can only take the corresponding discrete values. In particular the minimum non-zero value of $Q^{2}$ is given by $Q_{\min }^{2}=2 m_{\pi}\left(\sqrt{m_{\pi}^{2}+(2 \pi / L)^{2}}-\right.$ $m_{\pi}$ ), which for the parameters of our simulation is about $0.15 \mathrm{GeV}^{2}$. In this paper we study the form factor at small $Q^{2}$ (and in particular for $Q^{2} \ll 0.15 \mathrm{GeV}^{2}$ ), using the new technique proposed in [2] which allows one to carry out lattice computations at arbitrarily small values of $Q^{2}$. We now briefly review this technique.

In order to reach small momentum transfers, we use partially twisted boundary conditions $[5,6]$, combining gauge field configurations generated with sea quarks obeying periodic boundary conditions with valence quarks with twisted boundary conditions [5-14]. The valence quarks satisfy

$$
q\left(x_{k}+L\right)=e^{i \theta_{k}} q\left(x_{k}\right), \quad(k=1,2,3),
$$

where $q$ represents one of the degenerate up or down quarks. We have demonstrated in section 2.3 of ref. [2] that it is possible to introduce twisted boundary conditions independently for the three valence quarks and antiquarks, i.e. $\vec{\theta}_{1}$ for $q_{1}, \overrightarrow{\theta_{2}}$ for $q_{2}$ and $\overrightarrow{\theta_{3}}$ for $q_{3}$ in fig. 1. In our study it will be sufficient to set $\vec{\theta}_{3}=0$ so that the spectator quark or antiquark satisfies periodic boundary conditions. By varying $\vec{\theta}_{1}$ and $\vec{\theta}_{2}$ we are able to tune the momenta of the initial and final pions continuously.

The dispersion relation for a meson with twisting angle $\vec{\theta}$ takes the form $[8,10]$,

$$
E_{\pi}=\sqrt{m_{\pi}^{2}+\left(\vec{p}_{\vec{n}}+\frac{\vec{\theta}}{L}\right)^{2}},
$$

where $m_{\pi}$ is the pion mass and $\vec{p}_{\vec{n}}$ is the meson momentum induced by Fourier summation. For the matrix element in (2.1) with the initial and the final meson carrying momenta $\vec{p}=\vec{p}_{\vec{n}}+\vec{\theta} / L$ and $\vec{p}^{\prime}=\vec{p}_{\vec{n}^{\prime}}+\vec{\theta}^{\prime} / L$ respectively (where $\vec{\theta}=\vec{\theta}_{1}-\vec{\theta}_{3}$ and $\vec{\theta}^{\prime}=\vec{\theta}_{2}-\vec{\theta}_{3}$ ), the momentum transfer between the initial and the final state meson is

$$
q^{2}=\left(p-p^{\prime}\right)^{2}=\left(E_{\pi}(\vec{p})-E_{\pi}\left(\vec{p}^{\prime}\right)\right)^{2}-\left(\left(\vec{p}_{\vec{n}}+\vec{\theta} / L\right)-\left(\vec{p}_{\vec{n}^{\prime}}+\vec{\theta}^{\prime} / L\right)\right)^{2} .
$$


The finite-volume corrections with partially twisted boundary conditions decrease exponentially with $L$ similarly to those with periodic boundary conditions [5].

\section{The computation and results}

In this section we present the details of the computation of the electromagnetic form factor of a pion with mass $m_{\pi}=330 \mathrm{MeV}$. In the first subsection we explain which correlation functions are computed in order to be able to extract the form factor. The parameters of the simulations are presented in subsection 3.2 and a brief introduction to the use of noise-source propagators is given in subsection 3.3. In section 3.4 we present our results for the form factor. Finally in section 3.5 we compare the computational cost of computing correlation functions with point source and $\mathbb{Z}(2)$-wall source propagators.

\subsection{Correlation functions}

In order to determine the form factors we compute two- and three-point correlation functions. The two-point function is defined by

$$
C_{\pi}(t, \vec{p})=\sum_{\vec{x}} e^{i \vec{p} \cdot \vec{x}}\left\langle O_{\pi}(t, \vec{x}) O_{\pi}^{\dagger}(0, \overrightarrow{0})\right\rangle=\frac{\left|Z_{\pi}\right|^{2}}{2 E_{\pi}(\vec{p})}\left(e^{-E_{\pi}(\vec{p}) t}+e^{-E_{\pi}(\vec{p})(T-t)}\right),
$$

where $O_{\pi}=\bar{d} \gamma_{5} u$ is a local pseudoscalar interpolating operator for the pion. We have assumed that $t$ and $T-t$ (where $T$ is the temporal extent of the lattice) are sufficiently large for the correlation function to be dominated by the lightest state (i.e. the pion). The constant $Z_{\pi}$ is given by $Z_{\pi}=\left\langle\pi\left|O_{\pi}^{\dagger}(0, \overrightarrow{0})\right| 0\right\rangle$. The three-point function is defined by

$$
\begin{aligned}
C_{\pi \pi}\left(t, t_{f}, \vec{p}, \vec{p}^{\prime}\right) & =Z_{V} \sum_{\vec{x}_{f}, \vec{x}} e^{i \vec{p}^{\prime} \cdot\left(\vec{x}_{f}-\vec{x}\right)} e^{i \vec{p} \cdot \vec{x}}\left\langle O_{\pi}\left(t_{f}, \vec{x}_{f}\right) V_{4}(t, \vec{x}) O_{\pi}^{\dagger}(0, \overrightarrow{0})\right\rangle \\
& =\frac{Z_{V}\left|Z_{\pi}\right|^{2}}{4 E_{\pi}(\vec{p}) E_{\pi}\left(\vec{p}^{\prime}\right)}\left\langle\pi\left(\vec{p}^{\prime}\right)\left|V_{4}(0)\right| \pi(\vec{p})\right\rangle \\
& \times\left\{\theta\left(t_{f}-t\right) e^{-E_{\pi}(\vec{p}) t-E_{\pi}\left(\vec{p}^{\prime}\right)\left(t_{f}-t\right)}-\theta\left(t-t_{f}\right) e^{-E_{\pi}(\vec{p})(T-t)-E_{\pi}\left(\vec{p}^{\prime}\right)\left(t-t_{f}\right)}\right\},
\end{aligned}
$$

where $V_{4}$ is the time component of the bare electromagnetic current and where, without loss of generality, we have placed the source at the origin. Again we assume that all the time intervals in (3.2) are sufficiently large for the lightest hadrons to give the dominant contribution. As explained in the following paragraph, $Z_{V}$ is the normalization factor by which the bare lattice current needs to be multiplied in order to obtain the physical current.

The normalization factor $Z_{V}$ can readily be obtained as follows. For illustration we take $0<t<t_{f}<T / 2$, in which case $Z_{V}$ is defined by

$$
Z_{V}=\frac{\tilde{C}_{\pi}\left(t_{f}, \overrightarrow{0}\right)}{C_{\pi \pi}^{B}\left(t, t_{f}, \overrightarrow{0}, \overrightarrow{0}\right)}
$$

In the numerator we use the function $\tilde{C}_{\pi}(t, \vec{p})=C_{\pi}(t, \vec{p})-\frac{\left|Z_{\pi}\right|^{2}}{2 E_{\pi}(\vec{p})} e^{-E_{\pi}(\vec{p})(T-t)}$ where $Z_{\pi}$ and $E_{\pi}(\vec{p})=\sqrt{m_{\pi}^{2}+\vec{p}^{2}}$ are determined from fits to $C_{\pi}(t, \overrightarrow{0})$. For $t_{f}<T / 2$ this proves to be an 
effective and numerically stable procedure for the subtraction of the contribution from the backward propagating meson to $C_{\pi}\left(t_{f}, \overrightarrow{0}\right)$ in the numerator of $(3.3)$. (For $t_{f}=T / 2$ it is natural instead to use $\tilde{C}_{\pi}(t, \vec{p})=\frac{1}{2} C_{\pi}(t, \vec{p})$ in (3.3).) The superscript $B$ in the denominator indicates that we take the bare (unrenormalized) current in the three-point function.

In the following subsection we introduce the three datasets which we use for our analysis. For data set A we do not use twisted boundary conditions, setting $\vec{p}^{\prime}=0$ and determining the pion form factor from the ratio of correlation functions

$$
2 m_{\pi} \frac{C_{\pi \pi}\left(t, t_{f}, \vec{p}, \overrightarrow{0}\right)}{C_{\pi \pi}\left(t, t_{f}, \overrightarrow{0}, \overrightarrow{0}\right)} \frac{\tilde{C}_{\pi}(t, \overrightarrow{0})}{\tilde{C}_{\pi}(t, \vec{p})} \longrightarrow f^{\pi \pi}\left(q^{2}\right)\left(E_{\pi}(\vec{p})+m_{\pi}\right)
$$

For data sets $\mathrm{B}$ and $\mathrm{C}$ we use

$$
2 \sqrt{E_{\pi}(\vec{p}) E_{\pi}\left(\vec{p}^{\prime}\right)} \sqrt{\frac{C_{\pi \pi}\left(t, t_{f}, \vec{p}, \vec{p}^{\prime}\right) C_{\pi \pi}\left(t, t_{f}, \vec{p}^{\prime}, \vec{p}\right)}{\tilde{C}_{\pi}\left(t_{f}, \vec{p}\right) \tilde{C}_{\pi}\left(t_{f}, \vec{p}^{\prime}\right)}} \longrightarrow f^{\pi \pi}\left(q^{2}\right)\left(E_{\pi}(\vec{p})+E_{\pi}\left(\vec{p}^{\prime}\right)\right),
$$

(called ratio $R_{1}$ in [2]). Both ratios approach a constant for sufficiently large time intervals.

\subsection{Parameters of the simulation}

The computations described in this paper were performed using the ensemble with light quark mass $a m_{u}=a m_{d}=0.005$ and strange quark mass $a m_{s}=0.04$ from the set of $N_{f}=2+1$ flavour Domain Wall Fermion [15-17] configurations with $(L / a)^{3} \times T / a \times L_{s}=$ $24^{3} \times 64 \times 16$ which were jointly generated by the UKQCD/RBC collaborations using the QCDOC computer [18-21]. The bulk of the correlation functions were evaluated on the UK Research Councils' HECToR Cray XT4 computer, with the set completed using a University of Edinburgh BlueGene/L system as well as QCDOC. A detailed study of the light-hadron spectrum and other hadronic quantities using these configurations has recently been reported in ref. [1]. The gauge configurations were generated with the Iwasaki gauge action $[22,23]$ at $\beta=2.13$ corresponding to an inverse lattice spacing of $a^{-1}=$ $1.729(28) \mathrm{GeV}$. The resulting pion mass is $m_{\pi} \approx 330 \mathrm{MeV}$. In our numerical evaluations we use the masses measured directly on our data sets, which can be determined from the entries in tab. 2 and are fully consistent with the value reported in [1]. We use the jackknife technique to estimate the statistical errors.

In the following we distinguish three sets of correlation functions as specified in tab. 1. Set A was generated with point sources and sinks. We started the measurements for three different source positions on trajectories 900, 905 and 910, respectively, measuring on every 40th trajectory in each case and averaging three consecutive measurements over the sources into one bin. The initial pion carries momentum $|\vec{p}|=0, \frac{2 \pi}{L}$ or $\sqrt{2} \frac{2 \pi}{L}$ and the final pion is at rest. For this dataset we do not use twisted boundary conditions at all.

For data sets $\mathrm{B}$ and $\mathrm{C}$ we used a $\mathbb{Z}(2) \times \mathbb{Z}(2)$ noise wall source as explained in section 3.3 and a point sink. For data set $\mathrm{B}$ we started the measurement chains for the eight source positions specified in tab. 1 on trajectories $900,905,910, \ldots, 935$. Data set $\mathrm{C}$ is a subset of set B which starts with four source positions on trajectories 900,910, 920 and 930, respectively. In each case we measured on every 40th trajectory and averaged the correlation functions over the chains into bins of eight and four measurements, respectively. The 


\begin{tabular}{lcccc}
\hline \hline set & trajectories on $t_{\text {src }}=0$ & $\Delta$ & $N_{\text {meas }}$ & $t_{\text {src }}$ \\
\hline A & $900-4460$ & 20 & 537 & $0,16,32$ \\
B & $1000-6840$ & 40 & 1176 & $0,54,20,14,56,26,44,34$ \\
C & $1000-6440$ & 40 & 548 & $0,20,56,44$ \\
\hline \hline
\end{tabular}

Table 1: Details of measurements A, B and C. The quoted range of trajectories is the one for $t_{\mathrm{src}}=0$ and $\Delta$ is the separation in units of trajectories between subsequent measurements for each source position $t_{\mathrm{src}}$.

correlation functions obtained using sets B and C were computed with $\vec{p}_{\vec{n}}=\vec{p}_{\vec{n}^{\prime}}=0$ and the momenta of the initial and/or final pions were induced by twisting one of the pions' valence quarks. For each measurement we applied the full twist along one of the spatial directions. We changed this direction frequently as the measurements proceeded in order to reduce the correlations. In the cases in which both the initial and the final pion carried a twist, $\vec{\theta}$ and $\vec{\theta}^{\prime}$ were chosen to be anti-parallel.

Based on a preliminary study of a subset of data set A, we determined the pion mass $a m_{\pi}$ to have the central value 0.1907 and this guided us to choose twisting angles so as to obtain a suitable range of momentum transfers. (After a detailed analysis by the $\mathrm{RBC} / \mathrm{UKQCD}$ collaboration on their data set of choice, called the FPQ data set in [1], the mass was quoted as 0.1915(8).) For such a mass, as mentioned in section 2, the minimum value of $Q^{2}$ which can be reached without using twisted boundary conditions is $(a Q)_{\min }^{2} \approx 0.051\left(Q_{\min }^{2} \approx 0.152 \mathrm{GeV}^{2}\right)$. In order to reach smaller values of $Q^{2}$ we introduce three twisting angles $2.6832,2.1285$ and 1.6 , and in tab. 2 we summarize the corresponding kinematics.

\subsection{Three point functions from noise source propagators}

Lattice quark propagators are calculated by inverting the Dirac matrix $\mathcal{D}$ upon a matrix valued source $\eta$,

$$
S_{A, C}\left(t, \vec{x} ; t_{i}\right) \equiv \sum_{\vec{y}} \sum_{B} \mathcal{D}_{A, B}^{-1}\left(t, \vec{x} ; t_{i}, \vec{y}\right) \eta_{B, C}(\vec{y})
$$

where $A, B, C$ are spin-colour indices.

The hadronic form factor calculation is traditionally performed using point source propagators [2], for which the Dirac matrix is inverted from a single site with $\eta_{B, C}(\vec{y})=$ $\delta_{\vec{y}, \overrightarrow{0}} \delta_{B, C}$. However it has been shown $[4,24,25]$ that the use of stochastic sources allows for the calculation of meson propagators at a substantially reduced cost.

Following [24-26] we use source matrices with random elements from the set $\mathbb{Z}(2)$ for both real and imaginary components on a single source spin-colour index (0), for all sites $\vec{y}$ on the source timeslice: $\eta_{B, 0}\left(\vec{y}, t_{i}\right) \in \mathbb{Z}(2) \otimes \mathbb{Z}(2)$. With sources of this form, the solution $S\left(\vec{x}, t ; t_{i}\right)$ requires only a single inversion rather than the 12 required for the point solution. 


\begin{tabular}{cccccccc}
\hline \hline data set & $|\vec{p}| L$ & $\left|\vec{p}^{\prime}\right| L$ & $a E_{\pi}(\vec{p})$ & $a E_{\pi}\left(\vec{p}^{\prime}\right)$ & $(a Q)^{2}$ & $Q^{2}\left(\mathrm{GeV}^{2}\right)$ & $f^{\pi \pi}\left(q^{2}\right)$ \\
\hline $\mathrm{B}$ & 0 & 0 & $0.1910(4)$ & $0.1910(4)$ & 0 & 0 & 1 \\
$\mathrm{~B}$ & 0 & 1.6 & $0.1910(4)$ & $0.2023(4)$ & 0.004 & 0.013 & $0.9804(15)$ \\
$\mathrm{B}$ & 0 & 2.1285 & $0.1910(4)$ & $0.2106(4)$ & 0.007 & 0.022 & $0.9660(24)$ \\
$\mathrm{B}$ & 0 & 2.6832 & $0.1910(4)$ & $0.2213(4)$ & 0.012 & 0.035 & $0.9477(36)$ \\
$\mathrm{C}$ & 1.6 & 1.6 & $0.2023(6)$ & $0.2023(6)$ & 0.018 & 0.053 & $0.9189(75)$ \\
$\mathrm{C}$ & 2.1285 & 1.6 & $0.2106(5)$ & $0.2023(6)$ & 0.024 & 0.072 & $0.8943(88)$ \\
$\mathrm{C}$ & 2.1285 & 2.1285 & $0.2106(5)$ & $0.2106(5)$ & 0.031 & 0.094 & $0.867(10)$ \\
$\mathrm{C}$ & 2.6832 & 1.6 & $0.2213(5)$ & $0.2023(6)$ & 0.031 & 0.094 & $0.864(11)$ \\
$\mathrm{C}$ & 2.6832 & 2.1285 & $0.2213(5)$ & $0.2106(5)$ & 0.040 & 0.120 & $0.838(12)$ \\
$\mathrm{C}$ & 2.6832 & 2.6832 & $0.2213(5)$ & $0.2213(5)$ & 0.050 & 0.150 & $0.802(15)$ \\
\hline $\mathrm{A}$ & 0 & 0 & $0.1912(7)$ & $0.1912(7)$ & 0 & 0 & 1 \\
$\mathrm{~A}$ & $2 \pi$ & 0 & $0.3242(4)$ & $0.1912(7)$ & 0.051 & 0.152 & $0.809(14)$ \\
$\mathrm{A}$ & $\sqrt{2} 2 \pi$ & 0 & $0.4167(3)$ & $0.1912(7)$ & 0.086 & 0.258 & $0.711(26)$ \\
\hline \hline
\end{tabular}

Table 2: Table of accessible values of $Q^{2}=-q^{2}$ for the matrix element $\left\langle\pi\left(p^{\prime}\right)|V| \pi(p)\right\rangle$ together with the values of $f^{\pi \pi}\left(q^{2}\right)$. For data set $\mathrm{B}$ and $\mathrm{C}$ we also determined the correlation functions with momenta $|\vec{p}| L$ and $\left|\vec{p}^{\prime}\right| L$ interchanged.

A set $\left\{\eta^{j} \mid j=1, \ldots, N\right\}$ of these sources has the property that in the limit $N \rightarrow \infty$

$$
\frac{1}{N} \sum_{j=0}^{N} \eta_{A, 0}^{j}\left(\vec{x}, t_{i}\right) \eta_{0, B}^{\dagger j}\left(\vec{y}, t_{i}\right) \rightarrow \delta_{\vec{x}, \vec{y}} \delta_{A, B}
$$

such that the pseudoscalar two-point correlator at zero momentum tends to the spatial average of the point source solution $[24,25]$

$$
\begin{aligned}
C_{\pi}(t, \overrightarrow{0}) & =\sum_{j=0}^{N} \sum_{\vec{x}} \operatorname{tr}\left\{\gamma^{5} S^{j}\left(\vec{x}, t ; t_{i}\right) \gamma^{5}\left(\gamma^{5} S^{j}\left(\vec{x}, t ; t_{i}\right) \gamma^{5}\right)^{\dagger}\right\} \\
& \rightarrow \sum_{\vec{x}, \vec{y}} \operatorname{tr}\left\{\gamma^{5} \mathcal{D}^{-1}\left(\vec{x}, t ; \vec{y}, t_{i}\right) \gamma^{5}\left(\gamma^{5} \mathcal{D}^{-1}\left(\vec{x}, t ; \vec{y}, t_{i}\right) \gamma^{5}\right)^{\dagger}\right\}
\end{aligned}
$$

Although this explicitly projects to zero momentum at source, twisted boundary conditions can be used to induce a non-vanishing meson momentum.

The properties of equation (3.7) are retained on average when the sources $\eta^{i}$ reside on different configurations such that the stochastic sum can be included in the ensemble average. Therefore we require only a single stochastic source per configuration, giving an overall factor of 12 cost reduction over the traditional method.

This technique can be extended simply to three-point correlators using standard sequential source methods

$$
S^{\prime}\left(t_{i} ; t_{f}, \vec{p}_{f} ; t, \vec{x}\right)=\sum_{\vec{x}_{f}} \gamma_{5}\left(\mathcal{D}^{-1}\left(t, \vec{x} ; t_{f}, \vec{x}_{f}\right) \gamma^{5} S\left(t_{f}, \vec{x}_{f} ; t_{i}\right) e^{-i \vec{p}_{f} \cdot \vec{x}_{f}}\right)^{\dagger} \gamma_{5},
$$


the solution of which is again non-zero only on a single source spin-colour index, thus requiring only one extra inversion. The stochastic cancellation with the other source occurs at the source timeslice $t_{i}$ as in (3.8).

\subsection{Electromagnetic form factor of a pion with $m_{\pi}=330 \mathrm{MeV}$}

The main results of our computation, the form factor of a pion with $m_{\pi}=330 \mathrm{MeV}$ for a range of low values of $Q^{2}$ (obtained from all 3 data sets $\mathrm{A}, \mathrm{B}$ and $\mathrm{C}$ ), are presented in tab. 2 and plotted in fig. 2. The quoted energies have been determined using the continuum dispersion relation $E(\vec{p})=\sqrt{m_{\pi}^{2}+\vec{p}^{2}}$, where $m_{\pi}$ is the measured pion mass. We also show a zoom of the points at the lowest values of $Q^{2}$. The vertical dashed line represents the position of the lowest value of $Q^{2}$ which can be reached with periodic boundary conditions $\left(Q_{\min }^{2} \simeq 0.15 \mathrm{GeV}^{2}\right)$. From the figure it is satisfying to observe that at $Q_{\min }^{2}$ the results obtained with partially twisted boundary boundary conditions join smoothly onto the data obtained by performing a Fourier sum with momentum of magnitude $2 \pi / L$.

Our results from datasets $\mathrm{B}$ and $\mathrm{C}$ are well represented in the range $0 \leq Q^{2} \leq Q_{\text {min }}^{2}$ by the phenomenological pole formula

$$
f_{\text {pole }}^{\pi \pi}\left(q^{2}\right)=\frac{1}{1-q^{2} / M_{\text {pole }}^{2}} .
$$

From the slope of the form factor at $q^{2}=0$ we obtain the pion's electromagnetic charge radius, which is defined by

$$
\left.\left\langle r_{\pi}^{2}\right\rangle \equiv 6 \frac{d}{d q^{2}} f^{\pi \pi}\left(q^{2}\right)\right|_{q^{2}=0} .
$$

The best fit, which is shown as the blue curve in fig. 2 , gives $\left\langle r_{\pi}^{2}\right\rangle_{330 \mathrm{MeV}}=6 / M_{\text {pole }}^{2}=$ $0.382(37)(12)(15) \mathrm{fm}^{2}=0.382(42) \mathrm{fm}^{2}$, where the first error is statistical and the second is due to the uncertainty in the lattice spacing. The third error is to account for our lack of a continuum extrapolation (as discussed in sec. 4.2 below). This result corresponds to a pole mass of $\left(a M_{\text {pole }}\right)^{2}=0.202(20)$.

We compare our results to those of the UKQCD/QCDSF collaboration [27] who determined the pion form factor for a number of unphysical pion masses $m_{\pi} \geq 400 \mathrm{MeV}$ using periodic boundary conditions. For each pion mass, they fit their data to the pole form in (3.10) and hence determine the dependence of the pole mass $M_{\text {pole }}$ on the pion mass. Their results are well described by the ansatz,

$$
M^{2}\left(m_{\pi}^{2}\right)=c_{0}+c_{1} m_{\pi}^{2},
$$

for which they determined $c_{0}=0.517(23) \mathrm{GeV}^{2}$ and $c_{1}=0.647(30)$. Thus, for a pion of mass $330 \mathrm{MeV}$ they predict $\left\langle r_{\pi}^{2}\right\rangle_{330 \mathrm{MKV}}^{\mathrm{UKCD} / \mathrm{QCDSF}}=0.396(15) \mathrm{fm}^{2}$. This result, which we also illustrate in fig. 2, is compatible with ours.

Although the pole formula (3.10) is a good representation of our data for the full range $Q^{2} \leq Q_{\min }^{2}$, we find that the points at the smallest values of $Q^{2}$ tend to give a slightly smaller central value for the charge radius. We will take as our best estimates of $\left\langle r_{\pi}^{2}\right\rangle_{330 \mathrm{MeV}}$ the value obtained by applying $\mathrm{SU}(2) \mathrm{ChPT}$ to the points at small $Q^{2}$ as 

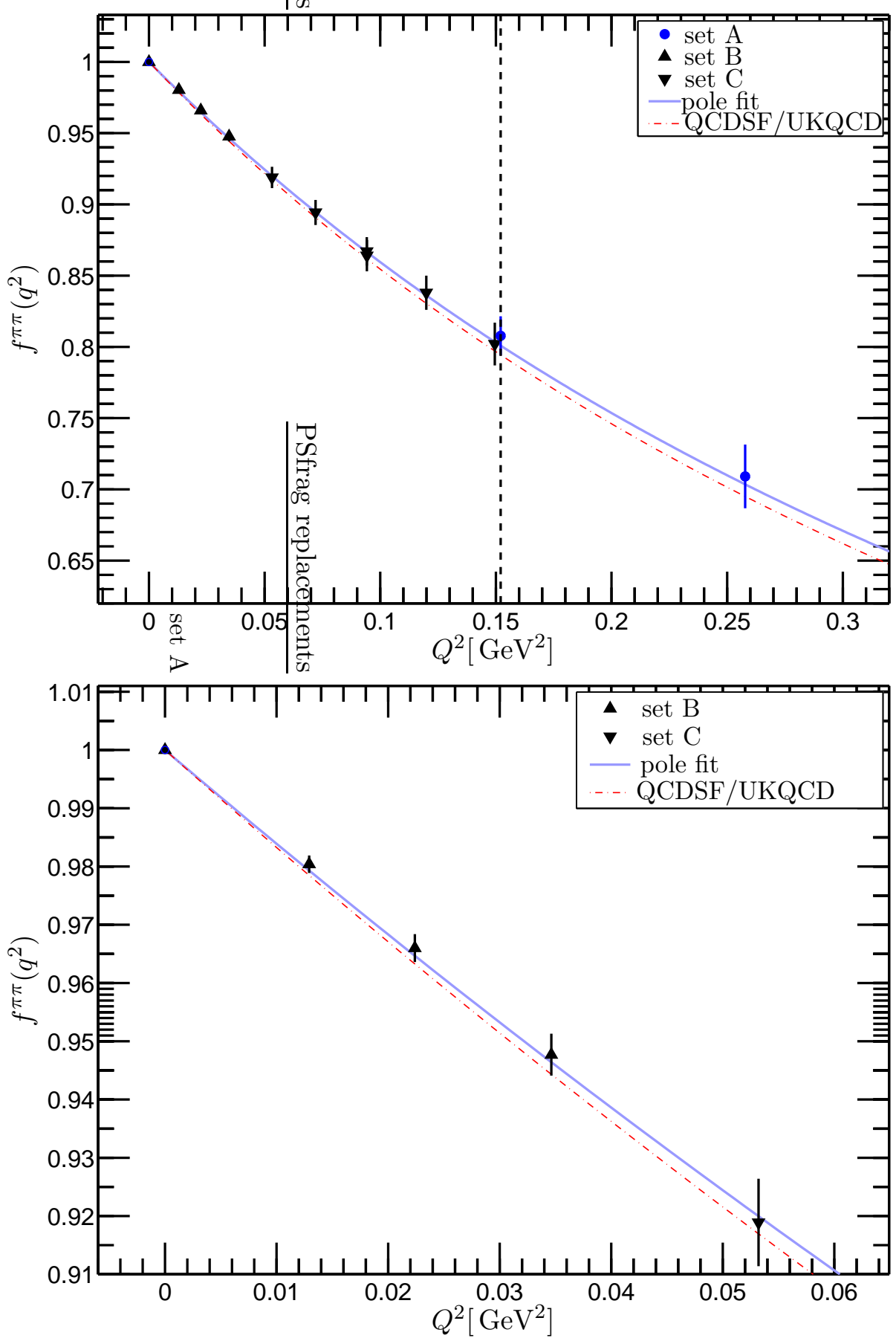

Figure 2: Results for the form factor $f^{\pi \pi}\left(q^{2}\right)$ for a pion with $m_{\pi}=330 \mathrm{MeV}$. The blue dashed curve is a pole fit to the data, while the red dashed curve shows the prediction for a $330 \mathrm{MeV}$ pion using results from the QCDSF/UKQCD collaboration [27]. The lower plot is a zoom into the very low $Q^{2}$ region.

explained in section 4 (we find $\left\langle r_{\pi}^{2}\right\rangle_{330 \mathrm{MeV}}=0.354(31) \mathrm{fm}^{2}$, see tab. 5 ). If we limit the fits to the points at small $Q^{2}$, the slope at $Q^{2}=0$ (and hence the charge radius) is not sensitive 


\begin{tabular}{cccccc}
\hline \hline data set & maximum $Q^{2}$ & linear & quadratic & cubic & pole \\
\hline B & $0.013 \mathrm{GeV}^{2}$ & $0.354(28)(11)$ & - & - & $0.361(29)(12)$ \\
B & $0.022 \mathrm{GeV}^{2}$ & $0.354(26)(11)$ & $0.353(35)(11)$ & - & $0.364(27)(12)$ \\
B & $0.035 \mathrm{GeV}^{2}$ & $0.353(25)(11)$ & $0.355(32)(11)$ & $0.351(41)(11)$ & $0.366(27)(12)$ \\
\hline C & $0.150 \mathrm{GeV}^{2}$ & $0.332(28)(11)$ & $0.387(44)(13)$ & $0.406(56)(13)$ & $0.382(37)(12)$ \\
\hline \hline
\end{tabular}

Table 3: Results for $\left\langle r_{\pi}^{2}\right\rangle_{330 \mathrm{MeV}}$ obtained by fitting to linear, quadratic or cubic functions of $Q^{2}$ and by using the pole ansatz (3.10). In the first row we use only the single point at the lowest value of $Q^{2}\left(Q^{2}=0.013 \mathrm{GeV}^{2}\right)$, in the second we use the two points at the lowest values of $Q^{2}$ $\left(Q^{2}=0.013 \mathrm{GeV}^{2}\right.$ and $\left.Q^{2}=0.022 \mathrm{GeV}^{2}\right)$ and in the third row we use the points at the lowest three values of $Q^{2}\left(Q^{2}=0.013 \mathrm{GeV}^{2}, Q^{2}=0.022 \mathrm{GeV}^{2}\right.$ and $\left.Q^{2}=0.035 \mathrm{GeV}^{2}\right)$. The final row corresponds to fits to all 9 points with $Q^{2} \leq Q_{\min }^{2}$. The two quoted errors are statistical and that due to the uncertainty in the lattice spacing.

to the precise form of the fitting function. To illustrate this we present in tab. 3 the results obtained by fitting our results for the form factor at the lowest three values of $Q^{2}$ to the pole form (3.10) as well as to linear, quadratic and cubic polynomials. In the final row of tab. 3 we present the value of $\left\langle r_{\pi}^{2}\right\rangle_{330 \mathrm{MeV}}$ obtained by applying the same fits to all 9 points up to $Q_{\min }^{2}$.

\subsection{Comparison of the cost of using point source and $\mathbb{Z}(2)$-wall source propa- gators}

In this study we have used two different formulations of the source in the computation of the quark propagators. The correlation functions on data set $\mathrm{A}$ have been computed from point source propagators while the correlation functions on data sets $\mathrm{B}$ and $\mathrm{C}$ have been computed using the noise source technique briefly described in section 3.3.

In this section we compare the relative computational cost of each approach in order to achieve similar statistical errors for standard observables relevant for the phenomenology of light mesons. In a very similar recent study [4] such a comparison was carried out for the meson spectrum on a $16^{3} \times 32$ lattice also using $N_{f}=2+1$ Domain Wall fermions and the Iwasaki gauge action. On this smaller volume the inverse lattice spacing was found to be $a^{-1}=1.63(3) \mathrm{GeV}$ and the study was performed using a pion with mass $a m_{\pi} \approx 0.44$. The statistical error on the pseudoscalar and vector meson correlation functions was studied at a fixed computational cost, i.e. at a fixed number of inversions of the Dirac matrix. It was found that the stochastic (one-end) approach offers a factor of two reduction in the error and a definite improvement in plateau quality over the traditional point source technique. Preliminary results indicating similar improvements were also reported by ETMC in [3]. Here we compare the costs for both approaches on a larger volume and for a much smaller pion mass. In particular we perform the comparison for $a m_{\pi}, Z_{V}$ and $f^{\pi \pi}\left(q^{2}=-Q_{\min }^{2}\right)$. Table 4 shows the results for each quantity for data sets $\mathrm{A}$ and $\mathrm{C}$. In the second column we give the number of inversions of the Dirac matrix that were carried out in each case. For one measurement 12 inversions are necessary in the case of point source propagators while only one inversion is necessary when using the noise source technique. On data set A we 


\begin{tabular}{ccccc}
\hline \hline data set & inversions & $m_{\pi}$ & $Z_{V}$ & $f^{\pi \pi}\left(-Q_{\min }^{2}\right)$ \\
\hline $\mathrm{A}$ & 6444 & $0.1912(7)$ & $0.7148(9)$ & $0.809(14)$ \\
$\mathrm{C}$ & 548 & $0.1910(6)$ & $0.7136(8)$ & $0.802(15)$ \\
\hline \hline
\end{tabular}

Table 4: Comparison of cost and error on quantities relevant for light meson phenomenology.

have $179 \times 12$ inversions times three for the number of sources. Our results for data sets A and $\mathrm{C}$ indicate that the same statistical error for $m_{\pi}, Z_{V}$ and $f^{\pi \pi}\left(-Q_{\min }^{2}\right)$ can be achieved with only about $1 / 12$ th of the computational cost when using the noise source technique. (This approximate gain of a factor of 12 found for this particular simulation should not be confused of course with the 12 inversions performed for each configuration and source for data set A.)

We have also tried to study the error for point-source and noise-source correlators at fixed cost, i.e. for a given number of inversions. The cost of the 1176 measurements which we carried out with the noise source (data set B) corresponds to $1176 / 12=98$ point source measurements. While we could carry out reliable fits to the correlators on data set B this was not the case for the sub-set of 98 measurements of data set A and no quantitative comparison seems possible. This observation shows however that the statistical properties of the correlation functions determined with noise-source propagators are better at the same computational cost.

Very light chiral quarks will display near zero modes associated with topological objects sampled in the ensemble. Intuitively, we might expect that point source propagators are more susceptible to the corresponding fluctuations, particularly if the location of the source is in the vicinity of such near zero modes. By contrast we expect such outliers to be averaged away when using a volume source like the one considered in this work. Using this picture it is not surprising that the gain observed in tab. 4 goes far beyond the one for the error of the pion mass observed in [4]. In that work the pion mass was more than double the one used here, thus the density of near zero modes was smaller. Furthermore the volume was $(2 / 3)^{3}$ of the one used here, allowing for less volume averaging in the case of the noise source.

\section{Electromagnetic form factor of a physical pion}

Having determined the electromagnetic form factor of a pion with $m_{\pi}=330 \mathrm{MeV}$ we can estimate what we would expect for that of a physical pion. The natural approach to perform this extrapolation is chiral perturbation theory and in the following subsection we briefly summarize the predictions of both the $\mathrm{SU}(2)_{\mathrm{L}} \times \mathrm{SU}(2)_{\mathrm{R}}$ and $\mathrm{SU}(3)_{\mathrm{L}} \times \mathrm{SU}(3)_{\mathrm{R}}$ theories.

\subsection{Chiral perturbation theory for the pion electromagnetic form factor}

The electromagnetic form factor of the pion has been studied extensively in both $\mathrm{SU}(2)_{\mathrm{L}} \times$ $\mathrm{SU}(2)_{\mathrm{R}}$ and $\mathrm{SU}(3)_{\mathrm{L}} \times \mathrm{SU}(3)_{\mathrm{R}}$ chiral perturbation theory $(\mathrm{ChPT})$. NLO expressions ap- 
pear in $[28,29]$ with extensions to NNLO in [30-32] and we now briefly summarise the results at NLO. NLO calculations in quenched ChPT and partially-quenched ChPT appear in [33]. An NLO calculation with partially twisted boundary conditions in partiallyquenched ChPT exists in [34]; this is particularly useful to estimate the finite-volume effects. In our lattice simulation we use unitary points (each valence quark mass is matched by a sea quark mass) and have small finite-volume effects (this will be justified below). We therefore use the continuum (unquenched) QCD results to obtain the form factor of the physical pion.

Current conservation ensures that $f^{\pi \pi}(0)=1$. At NLO only one low energy constant (LEC) is relevant for the form factor in both the $\mathrm{SU}(2)$ and $\mathrm{SU}(3)$ cases. This is denoted by $l_{6}^{r}(\mu)$ for $\mathrm{SU}(2)$ and $L_{9}^{r}(\mu)$ for $\mathrm{SU}(3)$ where the superscript $r$ stands for 'renormalised' and we have explicitly indicated the dependence on the renormalization scale $\mu$. The $\mathrm{SU}(2)$ [28] and $\mathrm{SU}(3)[29]$ expressions for the form factor are:

$$
\begin{aligned}
& f_{\mathrm{SU}(2), \mathrm{NLO}}^{\pi \pi}\left(q^{2}\right)=1+\frac{1}{f^{2}}\left[-2 l_{6}^{r} q^{2}+4 \tilde{\mathcal{H}}\left(m_{\pi}^{2}, q^{2}, \mu^{2}\right)\right] \\
& f_{\mathrm{SU}(3), \mathrm{NLO}}^{\pi \pi}\left(q^{2}\right)=1+\frac{1}{f_{0}^{2}}\left[4 L_{9}^{r} q^{2}+4 \tilde{\mathcal{H}}\left(m_{\pi}^{2}, q^{2}, \mu^{2}\right)+2 \tilde{\mathcal{H}}\left(m_{K}^{2}, q^{2}, \mu^{2}\right)\right]
\end{aligned}
$$

where

$$
\tilde{\mathcal{H}}\left(m^{2}, q^{2}, \mu^{2}\right)=\frac{m^{2} H\left(q^{2} / m^{2}\right)}{32 \pi^{2}}-\frac{q^{2}}{192 \pi^{2}} \log \frac{m^{2}}{\mu^{2}}
$$

and

$$
H(x) \equiv-\frac{4}{3}+\frac{5}{18} x-\frac{(x-4)}{6} \sqrt{\frac{x-4}{x}} \log \left(\frac{\sqrt{(x-4) / x}+1}{\sqrt{(x-4) / x}-1}\right)
$$

with $H(x)=-x / 6+O\left(x^{3 / 2}\right)$ for small $x$. For the space-like form factor considered in this paper $x=q^{2} / m^{2}$ is negative and $(x-4) / x>1$ so that the logarithm in (4.4) is real as expected. $f$ and $f_{0}$ are the pion decay constants in the $\mathrm{SU}(2)$ and $\mathrm{SU}(3)$ chiral limits respectively $\left(m_{u}=m_{d}=0\right.$ with $m_{s}$ at its physical value for $\mathrm{SU}(2)$ and $m_{u}=m_{d}=m_{s}=0$ for $\mathrm{SU}(3))$.

The NLO expressions for the charge radius are:

$$
\begin{aligned}
& \left\langle r_{\pi}^{2}\right\rangle_{\mathrm{SU}(2), \mathrm{NLO}}=-\frac{12 l_{6}^{r}}{f^{2}}-\frac{1}{8 \pi^{2} f^{2}}\left(\log \frac{m_{\pi}^{2}}{\mu^{2}}+1\right), \\
& \left\langle r_{\pi}^{2}\right\rangle_{\mathrm{SU}(3), \mathrm{NLO}}=\frac{24 L_{9}^{r}}{f_{0}^{2}}-\frac{1}{8 \pi^{2} f_{0}^{2}}\left(\log \frac{m_{\pi}^{2}}{\mu^{2}}+1\right)-\frac{1}{16 \pi^{2} f_{0}^{2}}\left(\log \frac{m_{K}^{2}}{\mu^{2}}+1\right) .
\end{aligned}
$$

Comparing the expressions for the charge radius gives the relation between the $\mathrm{SU}(2)$ and SU(3) NLO LEC's [29]:

$$
l_{6}^{r}(\mu)=-2 L_{9}^{r}(\mu)+\frac{1}{192 \pi^{2}}\left(\log \frac{\bar{m}_{K}^{2}}{\mu^{2}}+1\right),
$$

where $\bar{m}_{K}^{2}$ is the kaon mass in the chiral limit for the light quarks. Using the rho-mass for the renormalization scale, $\mu=m_{\rho}$, the second term on the right hand side of this relation is very small compared to the expected (power-counting) size of the LECs, so that 
$l_{6}^{r}\left(m_{\rho}\right) \approx-2 L_{9}^{r}\left(m_{\rho}\right)$. A word of caution should be added however. In deriving eq. (4.7) from eqs. (4.5) and (4.6) we have set $f_{0}=f$ which is correct at this order. In ref. [1] it was found that $f / f_{0} \simeq 1.23$ and so we may expect significant corrections to (4.7). We follow the approach of ref. [1] and use $\mathrm{SU}(2) \mathrm{ChPT}$ to obtain our best results.

The formulae above are obtained in infinite volume. Jiang and Tiburzi have used partially quenched, partially twisted $\mathrm{SU}(2)$ chiral perturbation theory to evaluate the finitevolume effects in the case where only one of the valence quarks is twisted [34]. This is the case for our three points at the lowest values of $Q^{2}\left(Q^{2}=0.013 \mathrm{GeV}^{2}, Q^{2}=0.022 \mathrm{GeV}^{2}\right.$, and $Q^{2}=0.035 \mathrm{GeV}^{2}$ ), which are the points which we use to determine the charge radius and the LECs $l_{6}^{r}$ and $L_{9}^{r}$. From figures 7 and 8 of [34] we see that for the pion mass $\left(m_{\pi}=330 \mathrm{MeV}\right)$ and volume, $(2.74 \mathrm{fm})^{3}$, used in our simulation, the finite volume effects in $\left\langle r_{\pi}^{2}\right\rangle$ and in $1-f^{\pi \pi}\left(q^{2}\right)$ are less than $1 \%$. Since the remaining errors quoted for these quantities for a pion with $m_{\pi}=330 \mathrm{MeV}$ are $7-8 \%$, we feel confident in neglecting the finite volume effects in the remainder of this analysis. In order to extend the calculations of ref. [34] to the case in which more than one of the valence quarks satisfies twisted boundary conditions we would have to perform SU(3) ChPT calculations in the partially quenched, partially twisted theory [2]; this is left for a future publication.

The recent QCDSF/UKQCD two-flavour results for the pion charge radius [27] include a larger systematic error, a downwards shift of $6-7 \%$, arising from finite volume effects. The estimate of these effects in [27] is obtained using a very different approach to the one we use. QCDSF/UKQCD fit form factor data from a range of lattice ensembles, each with a range of pion masses, to a pole form, eq. (3.10), with the pole mass given by eq. (3.12). A chirallyextrapolated value for the pole mass translates directly to the chirally-extrapolated result for the charge radius. Finite volume corrections are modeled by replacing the expression for the pole mass with

$$
M^{2}\left(m_{\pi}^{2}\right)=c_{0}+c_{1} m_{\pi}^{2}+c_{2} e^{-m_{\pi} L},
$$

where $L$ is the spatial extent of the lattice. For this second form, additional lattices with varying volumes are added to the fit, but the results for the lightest pion, $400 \mathrm{MeV}$, are omitted. A chiral and infinite volume extrapolation now yields a new physical charge radius, with the difference quoted as a finite volume systematic error.

We end this subsection with a discussion of another source of uncertainty which the use of chiral perturbation theory can help to estimate. The mass of the (sea) strange quark $\left(m_{s}\right)$ in the simulation is different from the physical one $\left(a m_{s}=0.04\right.$ in the simulation compared to the physical value 0.0343(16) found in ref. [1]). In $\mathrm{SU}(3) \mathrm{ChPT}$ we use the mass of the kaon as found from our simulation and hence obtain the value of the LEC $L_{9}^{r}$ without the need for further corrections. The LEC $l_{6}^{r}$ of SU(2) ChPT on the other hand depends on the mass of the strange quark and, since this is our preferred approach, we need to understand the amount by which $l_{6}^{r}$ could be shifted due to the different value of $m_{s}$. Using eq. (4.7) and the value of the mass from [1] to estimate $\bar{m}_{K}$, we find that the shift in $l_{6}^{r}\left(m_{\rho}\right)$ is about $0.9 \%$ and is hence negligible compared even to the $9 \%$ statistical error ( $11 \%$ total error) found in section 4.2 below (this is also the case if we use eqs. (4.5) and (4.6) without setting $f=f_{0}$, when the relative error grows to $1.3 \%$ ). For the remainder of 
the analysis we therefore neglect this uncertainty.

\subsection{Results for the physical pion}

ChPT describes the behaviour of the form factor as a function of both the momentum transfer and the quark masses, providing that these are sufficiently small. We fit our data at fixed quark masses (i.e. for the pion with mass $330 \mathrm{MeV}$ ) as a function of $q^{2}$ to the NLO formulae for both $\mathrm{SU}(2)$ and $\mathrm{SU}(3) \mathrm{ChPT}$, eqs. (4.1) and (4.2) respectively. In these fits we use the results $a f=0.0665(47)$ and $a f_{0}=0.0541(40)$ which were determined by the $\mathrm{RBC} / \mathrm{UKQCD}$ collaboration in [1] (in our normalization the decay constant of the physical pion is $\left.f_{\pi^{ \pm}}=130.7(4) \mathrm{MeV}\right)$. In this way we obtain the LECs $l_{6}^{r}$ and $L_{9}^{r}$. Having obtained the LECs in this way, we then use the ChPT formulae given above to determine the form factor (and hence the charge radius) of a physical pion $\left(m_{\pi}=139.57 \mathrm{MeV}[35]\right)$.

In ref. [1] it was found that whereas both $\mathrm{SU}(2)$ and $\mathrm{SU}(3)$ ChPT fit the data for the pion masses and decay constants, in the SU(3) case the NLO corrections were very large, particularly for the decay constant, casting doubt on the convergence of the chiral expansion. For this reason, in ref. [1] the main results were obtained using SU(2) ChPT and the above result for the decay constant in the chiral limit, af, includes both the statistical and systematic errors. The corresponding result for the decay constant in the SU(3) limit, $a f_{0}$, on the other hand, includes only the statistical error.

The results of the chiral extrapolation are summarized in tables 5 and 6 for the SU(2) and $\mathrm{SU}(3)$ cases respectively. In both tables the first column corresponds to the result of fitting only to the data point at our lowest value of $Q^{2}\left(Q^{2}=0.013 \mathrm{GeV}^{2}\right)$ to determine the single LEC $\left(l_{6}^{r}\left(m_{\rho}\right)\right.$ or $\left.L_{9}^{r}\left(m_{\rho}\right)\right)$ and the charge radius. In the second column we use the data points at the lowest two values of $Q^{2}\left(Q^{2}=0.013 \mathrm{GeV}^{2}\right.$ and $\left.0.022 \mathrm{GeV}^{2}\right)$ and in the final column we fit the data for the lowest three values of $Q^{2}$. The results in the three columns do not show any dependence on the chosen fit range at these small values of $Q^{2}$.

Our simulation was performed at a single value of the lattice spacing and we cannot extrapolate our results to the continuum limit. However, our action has $O\left(a^{2}\right)$ discretization errors and we follow [1] by assigning a systematic uncertainty of $4 \%$ to measured quantities, representing an estimate of $\left(a \Lambda_{\mathrm{QCD}}\right)^{2}$ for our lattice spacing. Thus we assign a $4 \%$ error from this source to our values for $1-f^{\pi \pi}\left(q^{2}\right)$. This relative error is propagated to our results for the LECs and $\left\langle r_{\pi}^{2}\right\rangle$, where it appears as the last error quoted in tables 5 and 6 .

Based on the experience of ref. [1] and because we only know the statistical error for $a f_{0}$, we take for our best estimate the result from the fit to the $\mathrm{SU}(2)_{\mathrm{L}} \times \mathrm{SU}(2)_{\mathrm{R}}$ expression at NLO including the three data points at $Q^{2}=0.013,0.022$ and $0.035 \mathrm{GeV}^{2}$,

$$
l_{6}^{r}\left(m_{\rho}\right)=-0.0093(10), \quad\left\langle r_{\pi}^{2}\right\rangle_{330 \mathrm{MeV}}=0.354(31), \quad\left\langle r_{\pi}^{2}\right\rangle_{\chi}=0.418(31) .
$$

Comparison of our values for $l_{6}^{r}\left(m_{\rho}\right)$ and $L_{9}^{r}\left(m_{\rho}\right)$ in tables 5 and 6 with the $\mathrm{SU}(2)-\mathrm{SU}(3)$ conversion formula in (4.7) reveals deviations up to around 50\%. By this we mean that the LECs obtained directly from the fit differ from the values extracted using the conversion formula with the other LEC as input. Large SU(3) NLO corrections were seen in the analysis in [1], and indeed the discrepancy can be reduced very significantly by using eqs.(4.5) and (4.6) without setting $f=f_{0}$. 


\begin{tabular}{lrrr}
\hline \hline$Q_{\max }^{2}\left[\mathrm{GeV}^{2}\right]$ & \multicolumn{1}{c}{0.013} & 0.022 & \multicolumn{1}{c}{0.035} \\
\hline $100 l_{6}^{r}\left(m_{\rho}\right)$ & $-0.932(79)(03)(63)(40)$ & $-0.933(73)(03)(63)(40)$ & $-0.932(71)(03)(63)(40)$ \\
$\left\langle r_{\pi}^{2}\right\rangle_{330 \mathrm{MeV}}\left[\mathrm{fm}^{2}\right]$ & $0.354(28)(12)(00)(14)$ & $0.354(26)(12)(00)(14)$ & $0.354(25)(12)(00)(14)$ \\
$\left\langle r_{\pi}^{2}\right\rangle_{\chi}\left[\mathrm{fm}^{2}\right]$ & $0.418(28)(12)(04)(14)$ & $0.419(26)(12)(04)(14)$ & $0.418(25)(12)(04)(14)$ \\
\hline \hline
\end{tabular}

Table 5: Results from the SU(2) ChPT fits. The errors are statistical, uncertainty in the lattice spacing, uncertainty in af and uncertainty from the continuum extrapolation respectively. The three columns correspond to using the data at the lowest, the lowest two and the lowest three nonzero values of $Q^{2}$ respectively, while $Q_{\max }^{2}$ denotes the largest value of $Q^{2}$ used in the determination.

\begin{tabular}{lccc}
\hline \hline$Q_{\max }^{2}\left[\mathrm{GeV}^{2}\right]$ & 0.013 & 0.022 & 0.035 \\
\hline $100 L_{9}^{r}\left(m_{\rho}\right)$ & $0.307(26)(03)(49)(13)$ & $0.308(24)(03)(49)(13)$ & $0.308(23)(03)(49)(13)$ \\
$\left\langle r_{\pi}^{2}\right\rangle_{330 \mathrm{MeV}}\left[\mathrm{fm}^{2}\right]$ & $0.354(28)(12)(00)(14)$ & $0.355(26)(12)(00)(14)$ & $0.355(25)(12)(00)(14)$ \\
$\left\langle r_{\pi}^{2}\right\rangle_{\chi}\left[\mathrm{fm}^{2}\right]$ & $0.460(28)(12)(16)(14)$ & $0.460(26)(12)(16)(14)$ & $0.460(25)(12)(16)(14)$ \\
\hline \hline
\end{tabular}

Table 6: Results from the $\mathrm{SU}(3) \mathrm{ChPT}$ fits. The errors are statistical, uncertainty in the lattice spacing, (statistical) uncertainty in $a f_{0}$ and uncertainty from the continuum extrapolation respectively. The three columns correspond to using the data at the lowest, the lowest two and the lowest three non-zero values of $Q^{2}$ respectively, while $Q_{\max }^{2}$ denotes the largest value of $Q^{2}$ used in the determination.

\begin{tabular}{|c|c|c|c|c|c|}
\hline \multicolumn{2}{|l|}{ collaboration } & \multicolumn{2}{|c|}{ technique } & $\left\langle r_{\pi}^{2}\right\rangle_{\chi}\left[\mathrm{fm}^{2}\right]$ & \multirow[b]{2}{*}{ 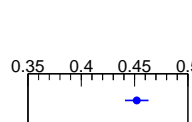 } \\
\hline PDG & [35] & & & $0.452(11)$ & \\
\hline Nam, Kim & [36] & instanton v & vacuum, large $N_{c}$ & 0.455 & - \\
\hline QCDSF/UKQCD & [27] & $N_{f}=2$ & Clover & $0.441(19)$ & - \\
\hline JLQCD & [37] & $N_{f}=2$ & Clover & $0.396(10)$ & - \\
\hline JLQCD & [38] & $N_{f}=2$ & Overlap & $0.388(15)$ & $\rightarrow$ \\
\hline $\mathrm{RBC} / \mathrm{UKQCD}$ & this work & $N_{f}=2+1$ & 1 Domain Wall & $0.418(31)$ & $\rightarrow$ \\
\hline
\end{tabular}

Table 7: Previous determinations (excluding quenched lattice results) of the pion's charge radius together with the value from the Particle data Group.

In tab. 7 we compare our result for the charge radius to the one determined from experiment and to other recent computations. Note that the previous lattice results were obtained with 2 flavours of sea quarks $\left(N_{f}=2\right)$ and using periodic boundary conditions so that the values of $Q^{2}$ are much larger than in this paper.

In fig. 3 we plot our lattice data for the $330 \mathrm{MeV}$ pion and the form factor of a physical pion obtained from this data using $\mathrm{SU}(2) \mathrm{ChPT}$. The experimental data from ref. [39] is also plotted together with the ChPT formula with the PDG world average for the charge radius (see also tab.7). 


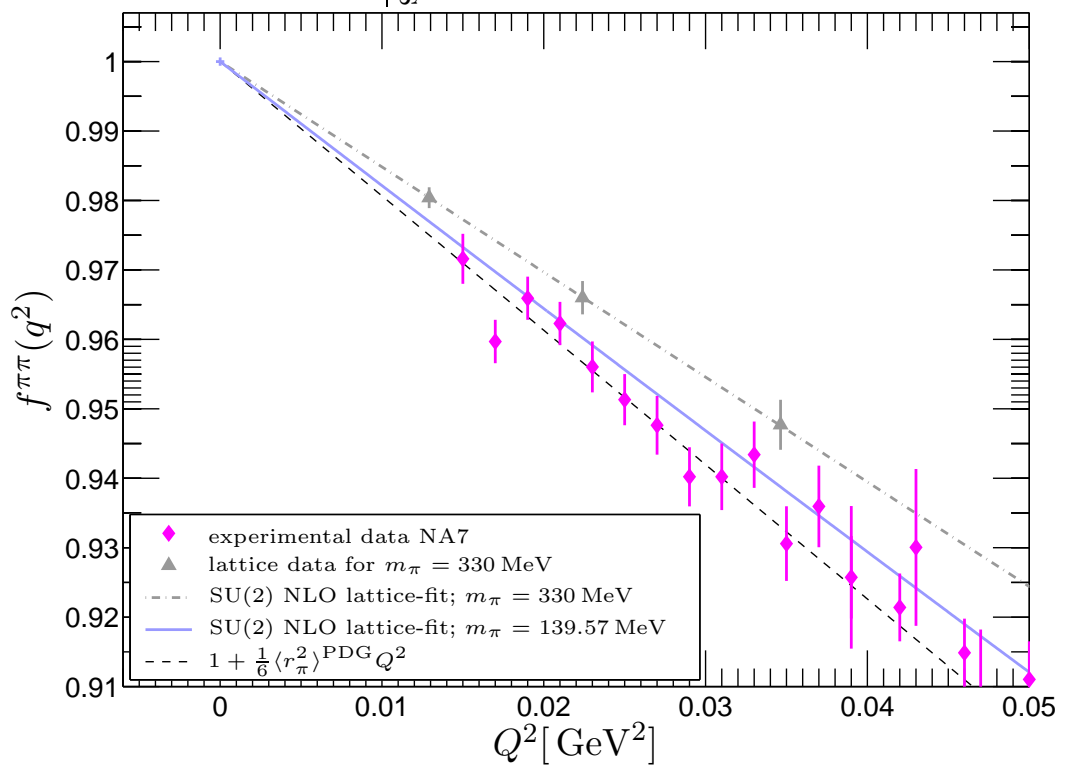

Figure 3: Comparison of experimental results (magenta diamonds) for the form factor $f^{\pi \pi}\left(q^{2}\right)$, lattice results at $m_{\pi}=330 \mathrm{MeV}$ (grey triangles and dash-dotted grey line) and the extrapolation of the lattice results to the physical point (blue solid line) using NLO SU(2) chiral perturbation theory. In addition we also represent the PDG world average for the charge radius using the black dashed line.

\section{Summary and conclusions}

In this paper we have successfully used partially twisted boundary conditions to compute the electromagnetic form factor of a pion with mass $330 \mathrm{MeV}$ at low values of $Q^{2}$. We use our results to compute the LEC $l_{6}^{r}$ of NLO SU(2) and then to determine the physical form factor and charge radius, see (1.1). We are able to calculate the form factor for values of $Q^{2}$ below the minimum value accessible with periodic boundary conditions, see fig. 2 . The results which we obtain are in good agreement with the experimentally determined form factor which gives us further confidence in the use of chiral perturbation theory in the mass range below $330 \mathrm{MeV}$ (indeed the value of $f$ which we use in the chiral extrapolation was obtained with pion masses up to $420 \mathrm{MeV}$ in ref. [1]). The techniques used in this paper can also be applied to other flavour non-singlet form factors of mesons and baryons and we strongly advocate the use of partially twisted boundary conditions in order to improve significantly the momentum resolution in lattice phenomenology.

One limitation of the current calculation of the pion's electromagnetic form factor is that it was performed at a single value of the lattice spacing, albeit with an action for which the discretization errors are of $O\left(a^{2}\right)$ and with good chiral and flavour properties. We are currently generating a set of configurations with the same action on a $32^{3}$ lattice with a finer lattice spacing and will repeat the present calculation with this ensemble. Although the mass and momentum transfers are sufficiently small to expect that NLO SU(2) ChPT is a good approximation, it would be nice to be able to check this explicitly. It is not clear whether in practice a full NNLO calculation can be performed with sufficient precision (i.e. 
whether the NNLO LECs will be determined sufficiently accurately) but, as it becomes possible to reach lighter quark masses, in the future we will be able to check the stability of the results. The finite-volume corrections for our mass and volume are small [34] and with our precision can be neglected.

In our calculation, we confirm the significant reduction in computational cost when computing three-point correlation functions using propagators computed from a single time-slice stochastic source compared to using point-source propagators.

\section{Acknowledgments}

We warmly thank Dirk Brömmel for informative discussions about the content of [27] and Bálint Joó for help in learning to use Cray XT4 systems. We are very grateful to the Engineering and Physical Sciences Research Council (EPSRC) for a substantial allocation of time on HECToR under the Early User initiative. We thank Arthur Trew, Stephen Booth and other EPCC HECToR staff for assistance and EPCC for computer time and assistance on BlueGene/L.

The calculations also made use of QCDOC computers, and we thank the support staff in the ACF at Edinburgh and at BNL. The QCDOC development and the resulting computer equipment used in this calculation were funded by the U.S. DOE grant DEFG02-92ER40699, PPARC JIF grant PPA/J/S/1998/00756 and by RIKEN. This work was supported by PPARC grants PPA/G/O/2002/00465, PP/D000238/1 and PP/C504386/1. Our calculations made use of the CHROMA [40] and BAGEL software packages.

JMF, AJ, HPdL and CTS acknowledge support from STFC Grant PP/D000211/1 and from EU contract MRTN-CT-2006-035482 (Flavianet). PAB, CK, CMM and JMZ acknowledge support from STFC grant PP/D000238/1.

\section{References}

[1] C. Allton et. al., Physical results from $2+1$ flavor domain wall QCD and $\mathrm{SU}(2)$ chiral perturbation theory, [arXiv:0804.0473].

[2] P. A. Boyle, J. M. Flynn, A. Jüttner, C. T. Sachrajda, and J. M. Zanotti, Hadronic form factors in lattice QCD at small and vanishing momentum transfer, JHEP 05 (2007) 016, [hep-lat/0703005].

[3] ETMC Collaboration, S. Simula, Pseudo-scalar meson form factors with maximally twisted Wilson fermions at $N_{f}=2$, [arXiv:0710.0097].

[4] P. A. Boyle, A. Juttner, C. Kelly, and R. D. Kenway, Use of stochastic sources for the lattice determination of light quark physics, [arXiv:0804.1501].

[5] C. T. Sachrajda and G. Villadoro, Twisted boundary conditions in lattice simulations, Phys. Lett. B609 (2005) 73-85, [hep-lat/0411033].

[6] P. F. Bedaque and J.-W. Chen, Twisted valence quarks and hadron interactions on the lattice, Phys. Lett. B616 (2005) 208-214, [hep-lat/0412023].

[7] P. F. Bedaque, Aharonov-Bohm effect and nucleon nucleon phase shifts on the lattice, Phys. Lett. B593 (2004) 82-88, [nucl-th/0402051]. 
[8] G. M. de Divitiis, R. Petronzio, and N. Tantalo, On the discretization of physical momenta in lattice QCD, Phys. Lett. B595 (2004) 408-413, [hep-lat/0405002].

[9] B. C. Tiburzi, Twisted quarks and the nucleon axial current, Phys. Lett. B617 (2005) 40-48, [hep-lat/0504002].

[10] UKQCD Collaboration, J. M. Flynn, A. Jüttner, and C. T. Sachrajda, A numerical study of partially twisted boundary conditions, Phys. Lett. B632 (2006) 313-318, [hep-lat/0506016].

[11] D. Guadagnoli, F. Mescia, and S. Simula, Lattice study of semileptonic form factors with twisted boundary conditions, Phys. Rev. D73 (2006) 114504, [hep-lat/0512020].

[12] G. Aarts, C. Allton, J. Foley, S. Hands, and S. Kim, Meson spectral functions at nonzero momentum in hot QCD, Nucl. Phys. A785 (2007) 202-205, [hep-lat/0607012].

[13] B. C. Tiburzi, Flavor twisted boundary conditions and isovector form factors, Phys. Lett. B641 (2006) 342-349, [hep-lat/0607019].

[14] T. B. Bunton, F. J. Jiang, and B. C. Tiburzi, Extrapolations of lattice meson form factors, Phys. Rev. D74 (2006) 034514, [hep-lat/0607001].

[15] D. B. Kaplan, A method for simulating chiral fermions on the lattice, Phys. Lett. B288 (1992) 342-347, [hep-lat/9206013].

[16] Y. Shamir, Chiral fermions from lattice boundaries, Nucl. Phys. B406 (1993) 90-106, [hep-lat/9303005].

[17] V. Furman and Y. Shamir, Axial symmetries in lattice QCD with Kaplan fermions, Nucl. Phys. B439 (1995) 54-78, [hep-lat/9405004].

[18] P. A. Boyle et. al., Overview of the QCDSP and QCDOC computers, IBM Journal of Research 49 (2005) 351-366.

[19] P. A. Boyle et. al., The QCDOC project, Nucl. Phys. Proc. Suppl. 140 (2005) 169-175.

[20] P. A. Boyle et. al., Hardware and software status of QCDOC, Nucl. Phys. Proc. Suppl. 129 (2004) 838-843, [hep-lat/0309096].

[21] QCDOC Collaboration, P. A. Boyle, C. Jung, and T. Wettig, The QCDOC supercomputer: Hardware, software, and performance, ECONF C0303241 (2003) THIT003, [hep-lat/0306023].

[22] Y. Iwasaki, Renormalization group analysis of lattice theories and improved lattice action: Two-dimensional nonlinear $O(N)$ sigma model, Nucl. Phys. B258 (1985) 141-156.

[23] Y. Iwasaki and T. Yoshié, Renormalization group improved action for SU(3) lattice gauge theory and the string tension, Phys. Lett. B143 (1984) 449.

[24] UKQCD Collaboration, M. Foster and C. Michael, Quark mass dependence of hadron masses from lattice qcd, Phys. Rev. D59 (1999) 074503, [hep-lat/9810021].

[25] UKQCD Collaboration, C. McNeile and C. Michael, Decay width of light quark hybrid meson from the lattice, Phys. Rev. D73 (2006) 074506, [hep-lat/0603007].

[26] S.-J. Dong and K.-F. Liu, Stochastic estimation with Z(2) noise, Phys. Lett. B328 (1994) 130-136, [hep-lat/9308015].

[27] QCDSF/UKQCD Collaboration, D. Brommel et. al., The pion form factor from lattice QCD with two dynamical flavours, Eur. Phys. J. C51 (2007) 335-345, [hep-lat/0608021]. 
[28] J. Gasser and H. Leutwyler, Chiral perturbation theory to one loop, Ann. Phys. 158 (1984) 142.

[29] J. Gasser and H. Leutwyler, Chiral perturbation theory: Expansions in the mass of the strange quark, Nucl. Phys. B250 (1985) 465.

[30] J. Bijnens, G. Colangelo, and P. Talavera, The vector and scalar form factors of the pion to two loops, JHEP 05 (1998) 014, [hep-ph/9805389].

[31] J. Bijnens, G. Colangelo, and G. Ecker, Renormalization of chiral perturbation theory to order $p^{6}$, Annals Phys. 280 (2000) 100-139, [hep-ph/9907333].

[32] J. Bijnens and P. Talavera, Pion and kaon electromagnetic form factors, JHEP 03 (2002) 046, [hep-ph/0203049].

[33] D. Arndt and B. C. Tiburzi, Charge radii of the meson and baryon octets in quenched and partially quenched chiral perturbation theory, Phys. Rev. D68 (2003) 094501, [hep-lat/0307003].

[34] F. J. Jiang and B. C. Tiburzi, Flavor twisted boundary conditions, pion momentum, and the pion electromagnetic form factor, Phys. Lett. B645 (2007) 314-321, [hep-lat/0610103].

[35] Particle Data Group Collaboration, W. M. Yao et. al., Review of particle physics, J. Phys. G33 (2006) 1-1232.

[36] S.-i. Nam and H.-C. Kim, Electromagnetic form factors of the pion and kaon from the instanton vacuum, [arXiv:0709.1745].

[37] JLQCD Collaboration, S. Hashimoto et. al., Pion form factors in two-flavor QCD, PoS LAT2005 (2006) 336, [hep-lat/0510085].

[38] JLQCD Collaboration, T. Kaneko et. al., Pion form factor from all-to-all propagators of overlap quarks, PoS LAT2007 (2007) 148, [arXiv:0710.2390].

[39] NA7 Collaboration, S. R. Amendolia et. al., A measurement of the space - like pion electromagnetic form-factor, Nucl. Phys. B277 (1986) 168.

[40] SciDAC Collaboration, R. G. Edwards and B. Joo, The Chroma software system for lattice QCD, Nucl. Phys. Proc. Suppl. 140 (2005) 832, [hep-lat/0409003]. 